\title{
Feature Optimization of Speech Emotion Recognition
}

\author{
Chunxia Yu, Ling Xie, Weiping $\mathrm{Hu}^{*}$ \\ GuangXi Key Lab of Multi-Source Information Mining and Security, GuangXi Normal University, Guilin, China \\ Email: ^huwp@mailbox.gxnu.edu.cn
}

How to cite this paper: Yu, C.X., Xie, L. and Hu, W.P. (2016) Feature Optimization of Speech Emotion Recognition. J. Biomedical Science and Engineering, 9, 37-43. http://dx.doi.org/10.4236/jbise.2016.910B005

Received: April 11, 2016

Accepted: September 20, 2016

Published: September 23, 2016

\begin{abstract}
Speech emotion is divided into four categories, Fear, Happy, Neutral and Surprise in this paper. Traditional features and their statistics are generally applied to recognize speech emotion. In order to quantify each feature's contribution to emotion recognition, a method based on the Back Propagation (BP) neural network is adopted. Then we can obtain the optimal subset of the features. What's more, two new characteristics of speech emotion, MFCC feature extracted from the fundamental frequency curve (MFCCF0) and amplitude perturbation parameters extracted from the shorttime average magnitude curve (APSAM), are added to the selected features. With the Gaussian Mixture Model (GMM), we get the highest average recognition rate of the four emotions $82.25 \%$, and the recognition rate of Neutral $90 \%$.
\end{abstract}

\section{Keywords}

Speech Emotion Recognition, Feature Selection, Feature Extraction, BP Neural Network, GMM

\section{Introduction}

Speech is one of the important ways for human's communication and it is a convenience and simple way to transmit information. The speech signal contains not only the expressed speech meaning, but also the speaker's emotion information which always be ignored by the traditionally speech processing [1]. But the emotion information plays a very important role in the speech communication. Therefore, in recent years, emotion recognition has become a hot spot. Traditional features, such as energy (E), zerocrossing rate (ZCR), the fundamental frequency (F0), the first formant (FF), Mel Frequency Cepstrum Coefficients (MFCC), linear prediction coefficient (LPC), the shorttime average magnitude (SAM) etc. and their statistics, such as maximum (Max), 
minimum (Min), mean, variance (Var.), first order difference (FOD), rate of change (RC) etc. are generally applied to recognize speech emotion. By combining the above features, we can use it to recognize a speech's emotion. In this paper, speech emotion is divided into four categories: Fear, Happy, Neutral and Surprise.

Due to the redundant and unrelated information between feature parameters, it is indispensable to select the features which remarkably characterize speech emotion information [2]. The paper adopts the Back Propagation (BP) neural network to sequence the features in the saliency measure and selects a set of features.

Based on the feature selection, two new characteristics of speech emotion, MFCC feature extracted from the fundamental frequency curve (MFCCF0) and amplitude perturbation parameters extracted from the short-time average magnitude curve (APSAM), are added to the selected features. The Gaussian Mixture Model (GMM) is used to recognize speech emotion [3]. According to the experiment result, the two new added features can effectively increase the recognition rate.

\section{Database}

A Chinese emotional database (CASIA) is used in this paper. CASIA was released by the Institute of Automation, Chinese Academy of Sciences [4]. It is composed of 1,200 wave files that represent different emotional states: happy, fear, sad, surprise, neutral and angry. Four actors (two females and two males) read 50 different texts respectively in the six emotions. Four kinds of emotions, Fear, Happy, Neutral and Surprise, 800 utterances in total (half of each emotion data to be trained, the remaining half to be tested) are chosen in the experiment.

\section{Feature Extraction}

\subsection{Traditional Features}

Traditional features are generally applied to recognize speech emotion which have proven to be useful [5]. In this section, first of all, we preprocess speech signals, such as pre-emphasis, framing (256 sampling points, 128 points frame-shift), adding-windows. After that we extract the feature parameters such as E, ZCR, F0, FF, MFCC, LPC from each frame signal and figure out their Max, Min, mean, Var., FOD. Moreover, there are two other features, the RC of F0, the RC of FF, 32 traditional features totally.

\subsection{New Features}

The method of speech emotion recognition based on the traditional features doesn't achieve good results. So we introduce two new features, MFCCF0 and APSAM, to improve the recognition rate.

We extract fundamental frequency parameters from each frame of speech signal by autocorrelation function method and obtain a fundamental frequency curve. Then median filtering of 5 points is adopted to smooth this curve and the points of fundamental frequency off tracking the curve would be deleted. At last we extract 4th order MFCC feature parameters from this processed curve which is our first new feature. 
Next we extract SAM of each frame of speech signal and achieve a SAM curve. On the basis of this curve, amplitude perturbation parameters, which is used to describe the jitter level within a certain range, can be figured out. Amplitude perturbation parameters, such as amplitude jitter percentage (Shim), amplitude jitter (ShdB), amplitude perturbation quotient (APQ), their formulas are as follows:

$$
\begin{aligned}
& \text { Shim }=\frac{1 /(N-1) \sum_{i=1}^{N-1}\left|A^{i}-A^{(i+1)}\right|}{1 / N \sum_{i=1}^{N-1} A^{i}} \\
& \text { ShdB }=\frac{1}{N-1} \sum_{i=1}^{N-1}\left|20 \log \left(A^{i+1} / A^{i}\right)\right| \\
& A P Q=\frac{1 /(N-1) \sum_{i=1}^{N-10}\left|1 / 10 \sum_{r=0}^{10} A^{(i+r)}-A^{(i+2)}\right|}{1 / N \sum_{i=1}^{N-1} A^{i}}
\end{aligned}
$$

where $A$ represents short-time average magnitude, $N$ represents numbers of short-time average magnitude, $i=1,2, \cdots, N$.

\section{Feature Selection Based on BP Neural Network}

In order to constitute the best set of features and reduce the dimensions of feature space, Ruck et al. come up with the sensitivity of the network outputs to its inputs which is used to rank the input features [6]. In the experiment, the network uses one hidden layer. The activating function of the hidden layer uses Sigmoid function, and the activating function of the output layer is a linear function. In the experiment, we let the number of the hidden nodes be 15 . The set of 32 traditional features is used as the network inputs. After the network has been trained, the weights in the network are determined. Then the saliency values for each input were calculated. As each network is started with a different set of random weights, we take 10 trained network's saliency values to an average in order to improve accuracy. After 10 experiments, we obtain the results of ranking the average saliency values, which is showed in Table 1 . The input with the highest saliency value, is ranked No.1 and the lowest is ranked No.32.

\section{Results and Discussion}

\subsection{Recognition Based on Traditional Features}

From the results (Table 1) of the saliency sequencing of 32 traditional features, we can see the first six traditional features arranged in descending order: E FOD, F0 mean, MFCC mean, ZCR FOD, LPC mean and E mean. We choose the first four, five, and six feature parameters respectively and recognize them with GMM. Recognition results are showed in Table 2, Table 3, and Table 4. For the different number of Gaussian mixture model (NGMM) results in considerable difference of the recognition rates, we experiment with many different numbers of Gaussian mixture model and show the most representative results as follows. 
Table 1. A rank of the 32 traditional features.

\begin{tabular}{cccccc}
\hline No. & Feature & No. & Feature & No. & Feature \\
\hline 1 & E FOD & 12 & F0 Var. & 23 & E Min \\
2 & F0 mean & 13 & E Max & 24 & MFCC Max \\
3 & MFCC mean & 14 & LPC FOD & 25 & LPC Max \\
4 & ZCR FOD & 15 & ZCR Max & 26 & F0 Min \\
5 & LPC mean & 16 & F0 RC & 27 & FF FOD \\
6 & E mean & 17 & FF Var. & 28 & FF RC \\
7 & FF Max & 18 & MFCC Var. & 29 & E Var. \\
8 & F0 FOD & 19 & ZCR Min & 30 & FF Min \\
9 & ZCR Var. & 20 & MFCC Min & 31 & MFCC FOD \\
10 & ZCR mean & 21 & LPC Var. & 32 & LPC Min \\
11 & FF mean & 22 & F0 Max & & \\
\hline
\end{tabular}

Table 2. Recognition rates of the first four features (E FOD, F0 mean, MFCC mean, ZCR FOD).

\begin{tabular}{|c|c|c|c|c|c|}
\hline \multirow{2}{*}{ NGMM } & \multicolumn{4}{|c|}{ Speech emotion recognition rate (\%) } & \multirow{2}{*}{ average } \\
\hline & Fear & Happy & Neutral & Surprise & \\
\hline 3 & 66 & 48 & 81 & 62 & 64.25 \\
\hline 4 & 78 & 70 & 69 & 75 & 73 \\
\hline 5 & 74 & 67 & 73 & 50 & 66 \\
\hline 6 & 79 & 71 & 69 & 69 & 72 \\
\hline 8 & 73 & 63 & 64 & 67 & 66.75 \\
\hline 12 & 69 & 54 & 66 & 67 & 64 \\
\hline
\end{tabular}

Table 3. Recognition rates of the first five features (E FOD, F0 mean, MFCC mean, ZCR FOD, LPC mean).

\begin{tabular}{|c|c|c|c|c|c|}
\hline \multirow{2}{*}{ NGMM } & \multicolumn{4}{|c|}{ Speech emotion recognition rate (\%) } & \multirow{2}{*}{ average } \\
\hline & Fear & Happy & Neutral & Surprise & \\
\hline 3 & 76 & 56 & 72 & 79 & 70.75 \\
\hline 4 & 82 & 73 & 85 & 75 & 78.75 \\
\hline 5 & 82 & 71 & 87 & 79 & 79.75 \\
\hline 6 & 76 & 73 & 88 & 74 & 77.75 \\
\hline 8 & 76 & 77 & 84 & 71 & 77 \\
\hline 12 & 69 & 65 & 82 & 70 & 71.5 \\
\hline
\end{tabular}

Table 4. Recognition rates of the first six features (E FOD, F0 mean, MFCC mean, ZCR FOD, LPC mean, E mean).

\begin{tabular}{|c|c|c|c|c|c|}
\hline \multirow{2}{*}{ NGMM } & \multicolumn{4}{|c|}{ Speech emotion recognition rate (\%) } & \multirow{2}{*}{ average } \\
\hline & Fear & Happy & Neutral & Surprise & \\
\hline 3 & 76 & 69 & 77 & 64 & 71.5 \\
\hline 4 & 77 & 72 & 86 & 78 & 78.25 \\
\hline 5 & 73 & 76 & 81 & 79 & 77.25 \\
\hline 6 & 69 & 77 & 87 & 80 & 78.25 \\
\hline 8 & 75 & 74 & 83 & 70 & 75.5 \\
\hline 12 & 60 & 59 & 84 & 74 & 69.25 \\
\hline
\end{tabular}


This paper only chooses the first few features used to recognize, because of their greater contribution to the speech emotion recognition. As show in the three tables above, when the first five features (E FOD, F0 mean, MFCC mean, ZCR FOD, LPC mean) are combined, the average recognition rate reaches the highest $79.75 \%$, in which the recognition rate of Fear reaches $82 \%$ and the recognition rate of Neutral reaches $87 \%$. If we continue to add emotional features as the inputs, we will find that the rate of single recognition and average recognition all decrease. This proved that the five features involve considerable information to differentiate emotions. With the increase of selected features, redundant and irrelevant between the features increase, and the recognition rates of the speech emotion decrease [7].

\subsection{Recognition Based on Traditional and New Features}

Based on the feature selection above, two new features of speech emotion, MFCCF0 and APSAM, are added to the selected features [8]. The recognition results of the first five features (E FOD, F0 mean, MFCC mean, ZCR FOD, LPC mean) and MFCCF0 are showed in Table 5. The recognition results of the first five features and APSAM are showed in Table 6. And the recognition results of the first five features, MFCCF0 and APSAM are showed in Table 7.

Table 5. Adding MFCCF0 based on the first five features.

\begin{tabular}{cccccc}
\hline \multirow{2}{*}{ NGMM } & \multicolumn{5}{c}{ Speech emotion recognition rate (\%) } \\
\cline { 2 - 5 } & Fear & Happy & Neutral & Surprise & \\
\hline 3 & 75 & 70 & 94 & 75 & 78.5 \\
4 & 85 & 73 & 90 & 74 & 80.5 \\
5 & 83 & 76 & 89 & 68 & 79 \\
6 & 74 & 73 & 87 & 75 & 77.25 \\
8 & 74 & 70 & 85 & 71 & 75 \\
12 & 73 & 64 & 81 & 76 & 73.5 \\
\hline
\end{tabular}

Table 6. Adding APSAM based on the first five features.

\begin{tabular}{cccccc}
\hline \multirow{2}{*}{ NGMM } & \multicolumn{5}{c}{ Speech emotion recognition rate (\%) } \\
\cline { 2 - 5 } & Fear & Happy & Neutral & Surprise & \\
\hline 3 & 68 & 73 & 78 & 59 & 69.5 \\
4 & 82 & 79 & 87 & 75 & 80.75 \\
5 & 84 & 75 & 86 & 62 & 76.75 \\
6 & 79 & 76 & 90 & 56 & 75.25 \\
8 & 76 & 70 & 88 & 76 & 77.5 \\
12 & 76 & 68 & 83 & 71 & 74.5 \\
\hline
\end{tabular}


Table 7. Adding MFCCF0 and APSAM based on the first five features.

\begin{tabular}{|c|c|c|c|c|c|}
\hline \multirow{2}{*}{ NGMM } & \multicolumn{4}{|c|}{ Speech emotion recognition rate (\%) } & \multirow{2}{*}{ average } \\
\hline & Fear & Happy & Neutral & Surprise & \\
\hline 3 & 75 & 68 & 72 & 81 & 74 \\
\hline 4 & 86 & 77 & 90 & 76 & 82.25 \\
\hline 5 & 84 & 75 & 85 & 81 & 81.25 \\
\hline 6 & 79 & 80 & 87 & 72 & 79.5 \\
\hline 8 & 82 & 73 & 86 & 77 & 79.5 \\
\hline 12 & 71 & 80 & 84 & 65 & 75 \\
\hline
\end{tabular}

As show in Table 5, after adding MFCCF0, the aver rate reaches $80.5 \%$ [9]. It is because of speech emotion is in relation to F0. While reading the different text in different emotions, their fundamental frequency curves are various. Also, emotion has nothing to do with the text. Therefore, feature parameters extracted from the fundamental frequency curve can characterize some emotion information and raise the recognition rate [10]. As show in Table 6, after adding APSAM, the aver rate increases by $1.0 \%$ and reaches $80.75 \%$. This is because of amplitude perturbation parameters can describe the jitter level within a certain range. Speech in different emotion causes different jitter level, so the feature could characterize emotional information to a certain extent and raise the recognition rates. From Table 7, we can see that, after adding the two new features, MFCCF0 and APSAM, the aver rate reaches $82.25 \%$, increases by $2.5 \%$, in which the Neutral gets $90 \%$ at its peak and the other three emotions all achieve $76 \%$ at least.

\section{Conclusion}

The method of feature selection based on BP neural network is not only convenient to choose the most effective ones in various traditional features, but also reduces the dimension of feature space [11]. The average rate reaches $79.75 \%$ while a set of 5 traditional features (E FOD, F0 mean, MFCC mean, ZCR FOD, LPC mean) is used to recognize speech emotion. Based on the feature selection, two new characteristics of speech emotion, MFCCF0 and APSAM, are added to the selected features (the first five features). With GMM, we get the highest average recognition rate of the four emotions $82.25 \%$, and the recognition rate of Neutral $90 \%$. According to the experiment result, the two new features can improve the recognition rate of speech emotion, because they can characterize some new emotion information.

\section{Acknowledgements}

This work was supported by the National Natural Science Foundation of China (No. 61062011, No. 61362003) and GuangXi Key Lab of Multi-source Information Mining \& Security, Electronic Engineering College, Guangxi Normal University. 


\section{References}

[1] Ayadi, M.E., Kamel, M.S. and Karray, F. (2011) Survey on Speech Emotion Recognition: Features, Classification Schemes, and Databases. Pattern Recog., 44, 572-587.

http://dx.doi.org/10.1016/j.patcog.2010.09.020

[2] Sultana, S., Shahnaz, C., Fattah, S.A., et al. (2014) Speech Emotion Recognition Based on Entropy of Enhanced Wavelet Coefficients. 2014 IEEE International Symposium on Circuits and Systems (ISCAS), Melbourne VIC, 1-5 June 2014, 137-140. http://dx.doi.org/10.1109/ISCAS.2014.6865084

[3] Zheng, W.Q., Yu, J.S. and Zou, Y.X. (2015) An Experimental Study of Speech Emotion Recognition Based on Deep Convolutional Neural Networks. 2015 International Conference on Affective Computing and Intelligent Interaction (ACII), Xi'an, 21-24 September 2015, 827-831. http://dx.doi.org/10.1109/ACII.2015.7344669

[4] Wang, K.X., An, N., Li, B.N., Zhang, Y.Y. and Li, L. (2015) Speech Emotion Recognition Using Fourier Parameters. IEEE Transactions on Affective Computing, 6, 69-75. http://dx.doi.org/10.1109/TAFFC.2015.2392101

[5] Bouwmans, T., Baf, F.E. and Vachon, B. (2008) Background Modeling Using Mixture of Gaussians for Foreground Detection. Recent Patents on Computer Science, 219-237. http://dx.doi.org/10.2174/2213275910801030219

[6] Ruck, D.W., Rogers, S.K. and Kabrisky, M. (1990) Feature Selection Using a Multilayer Perceptron. Journal of Neural Network Computing, 2, 40-48.

[7] Devijver, P.A. and Kittler, J. (1982) Pattern Recognition: A Statistical Approach. Prentice Hall International, London.

[8] Vlasenko, B., Schuller, B., Wendemuth, A. and Rigoll, G. (2007) Frame vs. Turn-Level: Emotion Recognition from Speech Considering Static and Dynamic Processing. Proceedings of the 2 nd International Conference on Affective Computing and Intelligent Interaction (ACII07), 139-147.

[9] Yuan, Y.J., Zhao, P.H. and Zhou, Q. (2010) Research of Speaker Recognition Based on Combination of LPCC and MFCC. Proc. IEEE Int. Conf. Intell. Comput. Intell. Syst., 3, 765-767. http://dx.doi.org/10.1109/icicisys.2010.5658337

[10] Li, Y. and Zhao, Y.X. (1998) Recognizing Emotions in Speech Using Short-Term and LongTern Features. Porc. ICSLP, Sydney, Australian, 2255-2258.

[11] Dellaert, F., Polzin, T. and Waibel, A. (1996) Recognizing Emotion in Speech. Proceedings of ICSLP, 1970-1973. http://dx.doi.org/10.1109/icslp.1996.608022 
Submit or recommend next manuscript to SCIRP and we will provide best service for you:

Accepting pre-submission inquiries through Email, Facebook, LinkedIn, Twitter, etc.

A wide selection of journals (inclusive of 9 subjects, more than 200 journals)

Providing 24-hour high-quality service

User-friendly online submission system

Fair and swift peer-review system

Efficient typesetting and proofreading procedure

Display of the result of downloads and visits, as well as the number of cited articles

Maximum dissemination of your research work

Submit your manuscript at: http://papersubmission.scirp.org/

Or contact jbise@scirp.org 